\title{
Clonal Deletion
}

National Cancer Institute

\section{Source}

National Cancer Institute. Clonal Deletion. NCI Thesaurus. Code C92166.

Processes that result in the removal of B and $T$ lymphocytes which have specificity for self antigens. 